\title{
Rheology of sheared flexible fiber suspensions via fiber-level simulations
}

\author{
Leonard H. Switzer III and Daniel J. Klingenberga) \\ Department of Chemical Engineering, University of Wisconsin-Madison, \\ Madison, Wisconsin 53706-1691
}

(Received 24 September 2002; final revision received 28 January 2003)

\begin{abstract}
Synopsis
We employ a particle-level simulation technique to investigate the rheology of non-Brownian, flexible fiber suspensions in simple shear flow. The model incorporates a variety of realistic features including fiber flexibility, fiber deformation, and frictional contacts. The viscosity of fiber suspensions is strongly influenced by the fiber equilibrium shape, interfiber friction, and fiber stiffness. The viscosity of the suspension increases as the fiber curvature, the coefficient of friction, or the fiber stiffness is increased. The yield stress of fiber suspensions scales with the volume fraction in a manner similar to that observed experimentally. Fiber suspensions that flocculate exhibit a shear thinning regime that extends to shear rates lower than those observed for homogeneous suspensions. (C) 2003 The Society of Rheology. [DOI: 10.1122/1.1566034]
\end{abstract}

\section{INTRODUCTION}

Suspensions of non-Brownian fibers are found in a variety of applications, such as pulp and paper and fiber-filled composite processing. Fiber suspensions exhibit nonNewtonian fluid characteristics similar to those of polymer melts and solutions, such as the Weissenberg effect (i.e., rod-climbing) [Nawab and Mason (1958); Mewis and Metzner (1974)], shear thinning [Goto et al. (1986); Kitano and Kataoka (1981)], and viscoelasticity [Wahren (1964); Thalén and Wahren (1964)]. Rheological properties, as well as other macroscopic quantities, depend on the structure of the suspensions. The structure is affected by such features as the fiber properties, interactions, suspending fluid properties, and the flow field imposed. By understanding the relationships among these features, the suspension structure and the macroscopic properties can therefore aid in the design and optimization of processes and products. In this article, we employ a simulation method to probe the relationships of fiber properties, interactions, suspension structure and the rheological properties of fiber suspensions in shear flow.

Adding fibers to a fluid can significantly alter its flow properties. Shear thinning of long fiber suspensions (aspect ratio $r_{p} \equiv L / d \geqslant 100$, where $L$ and $d$ are the fiber length and diameter, respectively) was reported by Kitano and Kataoka (1981) for vinylon fibers in silicone oil and by Goto et al. (1986) for nylon, glass, and vinylon fibers in glycerin. Goto et al. observed that shear thinning became more pronounced as the fiber aspect ratio increased or the flexibility increased (flexibility $\propto 1 / E_{Y} I$, where $E_{Y}$ is the fiber's Young's modulus and $I$ is the area moment of inertia). These researchers also reported differences in first normal stress that were similarly influenced by the fiber aspect ratio.

\footnotetext{
a) Author to whom all correspondence should be addressed; electronic mail: klingenberg@engr.wisc.edu
} 
The rheology of short glass fiber suspensions $\left(r_{p} \leqslant 50\right)$ was investigated by Petrich et al. (2000). The viscosity increased nearly linearly with the concentration, consistent with predictions for noninteracting fibers from slender body theory [Batchelor (1970a)]. Chaouche and Koch (2001) observed shear thinning for suspensions of short nylon fibers in various silicone oils at very low shear rates. They attributed the shear thinning behavior to adhesive contacts between fibers, and measured adhesive forces on the order of $0.01 \mu \mathrm{N}$. None of these researchers reported on the effect of fiber shape on rheological properties. Glass fibers tend to be straight, while nylon and vinylon fibers often have permanent deformation.

Meyer and Wahren (1964) proposed that sufficiently concentrated fiber suspensions form networks of contacting fibers that exhibit viscoelastic properties. Thalén and Wahren (1964) measured the shear modulus of pulp suspensions and obtained results in qualitative agreement with the simple network theory of Meyer and Wahren (1964). A sufficient force must be applied to the suspension to overcome the contact forces and cause the suspension to flow, and this behavior is characterized by yield stress. The yield stress of various wood and nylon fiber suspensions was measured by Bennington et al. (1990). The yield stress $\left(\sigma_{0}\right)$ scaled with the volume fraction $(\Phi)$ as $\sigma_{0} \sim \Phi^{\beta}$, where the exponent $\beta$ varied from 2.5 to 3.5. This is consistent with the simple fiber network theory of Meyer and Wahren (1964) which gives $\beta=3$. However, the measured values of $\beta$ varied with the fiber elasticity and aspect ratio, which is not predicted by simple network theory. The dependence of yield stress on the fiber elasticity was significantly different for suspensions of wood fibers and nylon fibers. Bennington et al. suggested the wood fibers have rougher surfaces than nylon which leads to different contact dynamics. Kitano and Kataoka (1981) also measured yield stresses for suspensions of vinylon fibers in silicone oil. The exponent $\beta$ decreased as the aspect ratio increased, which was attributed to fiber interactions, increased apparent flexibility, and wall effects.

Suspensions of flexible fibers often flocculate and form heterogeneous structures called flocs that affect the flow characteristics of the suspension. Soszynksi and Kerekes (1988) suggested that fiber flocs mainly form by mechanical contacts. They proposed a mechanism for fiber flocculation called "elastic fiber interlocking" in which fibers become locked in strained configurations due to their elasticity and friction forces at fiber contacts which serve to strengthen the network. Chen et al. (2002) measured the stress in wood and polyethylene terephthalate (PET) fiber suspensions that were observed to flocculate. At low shear rates, the suspensions behaved as Newtonian fluids. As the shear rate was increased, the suspensions would begin to flocculate, which corresponded to jumps in shear stress. As the shear rate was increased further, the flocs began to disappear and the fluid again exhibited Newtonian behavior.

The stress in a suspension of fibers is related to the distribution of fiber positions and orientations. The bulk average stress $\langle\sigma\rangle$ for a suspension of fibers in a Newtonian fluid is [Batchelor (1970b)]

$$
\langle\sigma\rangle \simeq-p \delta+2 \eta_{0} \mathbf{E}^{\infty}+\left\langle\sigma^{p}\right\rangle,
$$

where $p$ is an isotropic pressure, $\delta$ is the identity tensor, $\mathbf{E}^{\infty}$ is the rate of strain tensor of the flow field imposed, $\eta_{0}$ is the suspending fluid viscosity, and $\left\langle\sigma^{p}\right\rangle$ is the particle contribution to average stress. The particle contribution to stress can be divided into two parts: (1) particle-fluid interaction and (2) particle-particle interaction which results from nonzero hydrodynamic forces on the fibers. Batchelor (1970a) derived an expression for the particle stress using slender body theory for dilute suspensions of rigid, straight fibers that interact only via hydrodynamic disturbances, 


$$
\left\langle\sigma^{p}\right\rangle \approx \frac{\pi \eta_{0} n L^{3}}{6 \ln \left(2 r_{p}\right)}\left[\langle\mathbf{p p p p}\rangle-\frac{1}{3}\langle\mathbf{p p}\rangle \delta\right]: \mathbf{E}^{\infty},
$$

where $n$ is the number of fibers per unit volume, $L$ is the fiber length, and $\mathbf{p}$ is the unit fiber orientation vector. Slender-body theory can be used more generally to approximate the particle stress for suspensions of hydrodynamically interacting fibers into the semidilute regime $\left(n L^{3}>1\right)$,

$$
\left\langle\sigma^{p}\right\rangle=\frac{n}{2}\left\langle\int_{-\ell}^{\ell}\left[s \mathbf{p F}(s)+s \mathbf{F}(s) \mathbf{p}-\frac{2}{3}[s \mathbf{p} \cdot \mathbf{F}(s)] \delta\right] \mathrm{d} s+\mathbf{F}^{H} \mathbf{r}+\mathbf{r F}^{H}-\frac{1}{3}\left(\mathbf{F}^{H} \cdot \mathbf{r}\right) \delta\right\rangle,
$$

where $\mathbf{F}^{H}$ is the net hydrodynamic force on a fiber, $\mathbf{r}$ is the fiber's center of mass, and $\mathbf{F}(s)$ is the hydrodynamic force per unit length that acts on the fiber at axial position $s$. The quantity $\mathbf{F}(s)$ depends on the geometry of the particle, properties of the suspending fluid, and the orientation of the fibers in the flow field. The integral in Eq. (3) represents the purely hydrodynamic contribution to the particle stress [Mackaplow and Shaqfeh (1996)], and the remaining terms arise from nonhydrodynamic particle interactions [Sundararajakumar and Koch (1997)].

Particle-level simulations are a common method used to probe particulate suspensions and, more specifically, to understand the relationships of particle properties and interactions, the suspension microstructure, and macroscopic behavior [see, for example, Bossis and Brady (1987)]. The equations of motion for each particle are solved numerically, subject to the forces and torques identified, in order to evolve the particle positions and orientations over time and thus predict the suspension microstructure. This method is sufficiently general to allow the inclusion of a variety of features, such as elongated and flexible particles, as well as various forces, such as hydrodynamic forces and interactions, colloidal forces and friction, to name a few. The complexity of the physical model is only limited by the computational resources required to evaluate the forces and torques and to solve the equations of motion.

Numerous fiber suspension studies have focused on rigid, elongated bodies in Newtonian fluids. Claeys and Brady (1993a, 1993b) modeled fibers as rigid prolate spheroids (ellipsoids of revolution). They developed a method for accurately evaluating the hydrodynamic forces and torques, including both the short-range hydrodynamic interactions (lubrication forces) as well as the long-range, many-body hydrodynamic interactions. Mackaplow and Shaqfeh (1996) employed slender-body theory to accurately evaluate the long-range hydrodynamic interactions between prolate spheroids or cylinders. Like with the method of Claeys and Brady, the calculations were so computationally demanding that results for suspensions of long fibers in simple shear flow were limited to prescribed suspension structures. Thus these methods were not employed to predict the suspension structure resulting from flow. Simulations by Yamane et al. (1994) and by Fan et al. (1998) employed approximations for the hydrodynamic interactions between rigid fibers. These authors obtained good agreement with experimental results for the suspension viscosity as a function of the concentration, but did not report shear thinning behavior $\left(n L^{3} \lesssim 50, r_{p} \lesssim 30\right)$.

Sundararajakumar and Koch (1997) and Harlen et al. (1999) simulated suspensions of rigid, slender rods interacting via contact forces. They argued that for flowing suspensions of fibers, lubrication forces cannot prevent fibers from contacting, and thus shortrange hydrodynamic interactions were neglected. Harlen et al. (1999) simulated single spheres falling through neutrally buoyant fiber suspensions to illustrate the importance of 
fiber-fiber contacts on the flow properties of fiber suspensions. For low concentrations, interfiber contacts are rare and the flow behavior is dominated by long-range hydrodynamic interactions. However, as the concentration is increased such that the fibers are in frequent contact, the flow behavior is strongly influenced by the contacts. In fact, for $n L^{3} \geqslant 12\left(r_{p}=20\right)$, the drag on the settling sphere calculated by including long-range hydrodynamic interactions and contact forces is indistinguishable from that calculated by including contact forces alone [and in good agreement with experimental results reported by Milliken et al. (1989)]. Although the falling sphere influences the suspension structure, the authors did not report any tendency toward fiber flocculation.

Several studies have focused on simulating suspensions of flexible fibers. Yamamoto and Matsuoka $(1993,1994)$ modeled flexible fibers as chains of rigid spheres connected through springs, with potentials to mimic resistance to bending and twisting. Chain connectivity is maintained by constraints, thus producing equations that must be solved simultaneously with the equations of motion. Ross and Klingenberg (1997) modeled flexible fibers as inextensible chains of rigid prolate spheroids connected through ball and socket joints. This model eliminates the need for iterative constraints to maintain fiber connectivity, and can represent large aspect ratio fibers with relatively few bodies. These features help to reduce computations and facilitate simulation of concentrated suspensions. Schmid et al. (2000) extended this method by modeling flexible fibers as chains of spherocylinders connected by ball and socket joints that interact via short-range repulsive forces as well as friction forces. They demonstrated that the fiber equilibrium shape, flexibility, and interfiber friction are important in determining the suspension microstructure, and in particular for predicting flocculation behavior.

In this work, we build on the method of Schmid et al. to investigate relationships between fiber properties and interactions, and the resulting suspension rheological properties for simple shear flow. In Sec. II, we briefly describe the flexible fiber model and simulation method. The model fiber consists of rigid spherocylinders connected by ball and socket joints. The fibers interact with other fibers via short-range repulsive forces and friction. Fiber and suspension characteristics such as the equilibrium shape, flexibility, aspect ratio, friction, concentration, and suspending fluid characteristics have been shown to impact the structure of fiber suspensions in simulations [Schmid et al. (2000); Switzer (2002)]. Varying these parameters can result in dramatic changes to the suspension structure including the formation of heterogeneities (i.e., flocs). In Sec. III, we show that suspension rheological properties also depend sensitively on these features. The dependence of the shear thinning behavior and first normal stress difference on the fiber aspect ratio is similar to that observed in experiments. Calculated yield stresses exhibited scaling with the concentration in agreement with network theory and experiment. Fiber suspensions were also seen to flocculate under certain conditions, and this affected the shear thinning behavior of the suspensions.

\section{SIMULATION METHOD}

Flexible fiber suspensions are modeled as chains of neutrally buoyant, linked rigid bodies immersed in a Newtonian liquid. The model includes realistic features such as fiber flexibility, deformed equilibrium shapes, and mechanical contact forces between fibers. The model and simulation method are similar to that employed by Schmid et al. (2000) and is described in more detail elsewhere [Switzer (2002)].

Each fiber in the suspension is represented by $N_{\text {seg }}$ rigid cylinders (length $2 \ell$, radius $b$ ) with hemispherical end caps, connected end-to-end by ball and socket joints (Fig. 1). The motion of the fiber segments is described by Newton's laws of motion in which we 


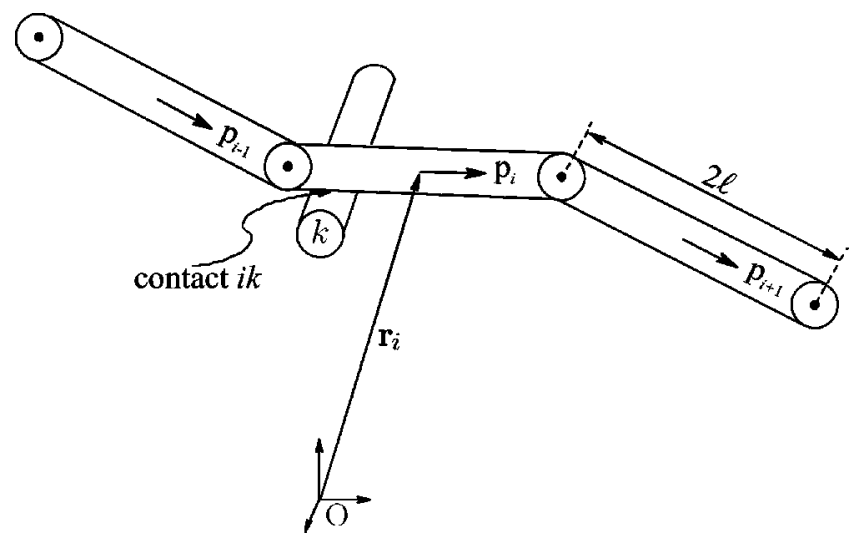

FIG. 1. Model fiber of rigid spherocylinders linked by ball and socket joints that experience mechanical contact with other fibers in the suspension.

neglect fiber inertia. The force balance on a fiber segment $i$ includes contributions from hydrodynamic drag $\left(\mathbf{F}_{i}^{\text {hyd }}\right)$, mechanical contact forces $\left(\mathbf{F}_{i k}^{\text {con }}\right)$, and forces at each joint that keep the fiber at a constant length $\left(\mathbf{X}_{i}\right)$,

$$
\mathbf{F}_{i}^{\mathrm{hyd}}+\mathbf{X}_{i+1}-\mathbf{X}_{i}+\sum_{k}^{N_{C_{i}}} \mathbf{F}_{i k}^{\mathrm{con}}=0
$$

where $N_{C_{i}}$ is the number of contacts on fiber segment $i$. The torque balance on fiber segment $i$ includes similar contributions with the addition of a restoring torque at each joint $\left(\mathbf{Y}_{i}\right)$,

$$
\mathbf{T}_{i}^{\mathrm{hyd}}+\mathbf{Y}_{i+1}-\mathbf{Y}_{i}+\ell \mathbf{p}_{i} \times\left[\mathbf{X}_{i+1}+\mathbf{X}_{i}\right]+\sum_{k}^{N_{C_{i}}}\left[\mathbf{G}_{i k} \times \mathbf{F}_{i k}^{\mathrm{con}}\right]=0,
$$

where $\mathbf{T}_{i}^{\text {hyd }}$ is the hydrodynamic torque, $\mathbf{p}_{i}$ is the orientation vector of the segment, and $\mathbf{G}_{i k}$ is a vector from the center of segment $i$ to the point of contact with segment $k$.

In this model, hydrodynamic interactions between fibers are neglected based on results reported by Sundararajakumar and Koch (1997) and by Harlen et al. (1999), as previously explained. We also ignore hydrodynamic interactions between segments within a fiber. These assumptions allow us to evaluate the hydrodynamic force and torque on a segment as that on an isolated body, $\mathbf{F}_{i}^{\text {hyd }}=\mathbf{A}_{i} \cdot\left[\mathbf{U}_{i}^{\infty}-\dot{\mathbf{r}}_{i}\right]$ and $\mathbf{T}_{i}^{\text {hyd }}=\mathbf{C}_{i} \cdot\left[\boldsymbol{\Omega}_{i}^{\infty}-\omega_{i}\right]$ $+\tilde{\mathbf{H}}_{i}: \mathbf{E}^{\infty}$, where the resistance tensors $\mathbf{A}_{i}, \mathbf{C}_{i}$, and $\tilde{\mathbf{H}}_{i}$ are approximated by the resistance tensors of a prolate spheroid with an equivalent aspect ratio, $r_{e_{s}}=0.7 r_{p_{s}}$ [Schmid et al. (2000); Switzer (2002); Kim and Karrila (1991)], where $r_{p_{s}}$ is the aspect ratio of a segment. The error associated with neglecting hydrodynamic interactions between segments is discussed in the Appendix. The ambient velocity, angular velocity, and rate of strain tensor are $\mathbf{U}_{i}^{\infty}, \boldsymbol{\Omega}_{i}^{\infty}$, and $\mathbf{E}^{\infty}$, respectively, and only simple shear flows are simulated [i.e., $\left.\mathbf{U}^{\infty}=(\dot{\gamma} z, 0,0)\right]$. The segment translational and angular velocities are $\dot{\mathbf{r}}_{i}$ and $\boldsymbol{\omega}_{i}$.

The restoring torque $\mathbf{Y}_{i}$ describes the resistance of the elastic fibers to bending and twisting. The bending and twisting components of this torque are assumed to be linear in 
the difference between the bending and twisting angles ( $\theta_{i}$ and $\phi_{i}$, respectively) and their equilibrium values $\left(\theta_{i}^{\mathrm{eq}}\right.$ and $\left.\phi_{i}^{\mathrm{eq}}\right)$,

$$
\left|\mathbf{Y}_{i}\right|=\kappa_{b}\left(\theta_{i}-\theta_{i}^{\mathrm{eq}}\right)+\kappa_{t}\left(\phi_{i}-\phi_{i}^{\mathrm{eq}}\right),
$$

where $\kappa_{b}$ and $\kappa_{t}$ are the bending and twisting constants of the fiber. The bending constant is related to the fiber stiffness $E_{Y} I$ by $\kappa_{b}=E_{Y} I / 2 \ell$, where $E_{Y}$ is the Young's modulus, $I \equiv \pi b^{4} / 4$ is the area moment, and $\ell$ is the half length of a fiber segment. The twisting constant is set to $\kappa_{t}=0.67 \kappa_{b}$ in this study, equal to that of an elastic circular cylinder with a Poisson ratio of 0.5 . The fiber flexibility is characterized by a single parameter which we call the effective stiffness $S^{\text {eff }} \equiv E_{Y} I / \eta_{0} \dot{\gamma} L^{4}$, where $\eta_{0}$ is the suspending fluid viscosity, $\dot{\gamma}$ is the shear rate, and $L$ is the total length of the fiber. The effective stiffness characterizes the relative importance of fiber stiffness and hydrodynamic torque in determining the amount a fiber will bend in shear flow. As $S^{\text {eff }} \rightarrow 0$, fibers behave like completely flexible threads, whereas for $S^{\text {eff }} \rightarrow \infty$, fibers become rigid and retain their equilibrium shape during flow.

The fiber length is kept constant by applying a constraint for each joint,

$$
\mathbf{r}_{i}+\ell \mathbf{p}_{i}=\mathbf{r}_{i+1}-\ell \mathbf{p}_{i+1},
$$

where $\mathbf{r}_{i}$ is the position of the segment. These constraint equations allow the solution of the constraint forces at each joint $\mathbf{X}_{i}$.

Fibers experience mechanical contacts with other fibers in the suspension. The force that results from each contact is decomposed into two components-a force in the normal direction of contact $\left(\mathbf{F}_{i k}^{\mathrm{N}}\right)$ and a frictional force $\left(\mathbf{F}_{i k}^{\text {fric }}\right)$ in the plane of contact. The purely repulsive normal force exerted on segment $i$ by segment $k$ is modeled as $\mathbf{F}_{i k}^{\mathrm{N}}$ $=-F \exp \left[-a h_{i k}\right] \mathbf{n}_{i k}$, where $h_{i k}$ is the separation between the surfaces of segments $i$ and $k, \mathbf{n}_{i k}$ is the unit normal vector directed from segment $i$ to $k, F=900 \pi \eta_{0} \ell b \dot{\gamma}$ is the magnitude of the repulsive force, and $a=20 / b$. The friction force is determined by the constraint of no relative motion in the plane of contact,

$$
\left(\begin{array}{c}
\Delta \mathbf{u}_{i k} \cdot \mathbf{e}_{1}^{\text {loc }} \\
\Delta \mathbf{u}_{i k} \cdot \mathbf{e}_{2}^{\text {loc }} \\
\mathbf{F}_{i k}^{\text {fric }} \cdot \mathbf{n}_{i k}
\end{array}\right)=0,
$$

where $\Delta \mathbf{u}_{i k}$ is the relative velocity between segments $i$ and $k$ at the point of contact, and the plane of contact is defined by the vectors $\mathbf{e}_{1}^{\text {loc }}$ and $\mathbf{e}_{2}^{\text {loc }}$. The calculated friction force is then subjected to a Coulombic friction law of the form

$$
\begin{aligned}
\left|\mathbf{F}_{i k}^{\text {fric }}\right| & \leqslant \mu^{\text {stat }}\left|\mathbf{F}_{i k}^{\mathrm{N}}\right| \Rightarrow \text { contact remains intact, } \\
& >\mu^{\text {stat }}\left|\mathbf{F}_{i k}^{\mathrm{N}}\right| \Rightarrow \mathbf{F}_{i k}^{\text {fric }}=\mu^{\text {kin }}\left|\mathbf{F}_{i k}^{\mathrm{N}}\right| \frac{\Delta \mathbf{u}_{i k}}{\left|\Delta \mathbf{u}_{i k}\right|},
\end{aligned}
$$

where $\mu^{\text {stat }}$ and $\mu^{\text {kin }}$ are the static and kinetic coefficients of friction. For all of the simulation results presented in this article, the kinetic coefficient of friction is $\mu^{\text {kin }}$ $=0$ (i.e., no sliding friction).

The equations of motion and the constraint equations for all of the fiber segments in the suspension can be expressed as a system of differential algebraic equations (DAEs) for the unknown coordinates and constraint forces,

$$
\dot{\mathbf{q}}-\mathbf{F}(\mathbf{q}, \boldsymbol{\lambda})=\mathbf{0},
$$



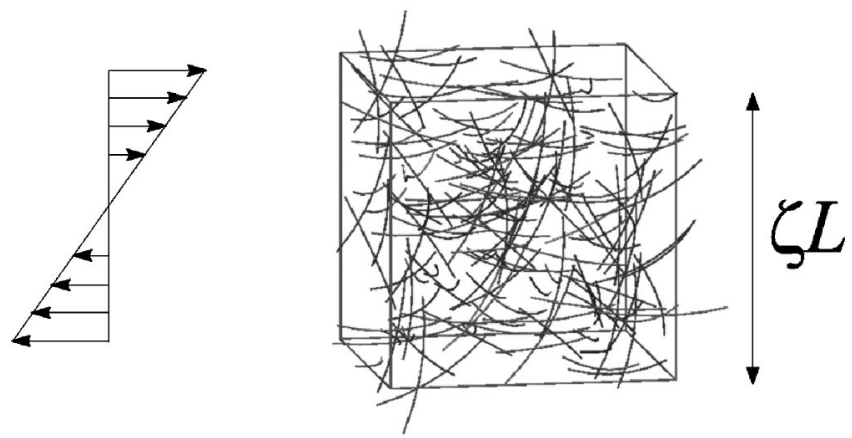

FIG. 2. Example of the starting configuration of fibers randomly placed in a periodic simulation cell.

$$
\begin{gathered}
\boldsymbol{\Psi}(\mathbf{q})=\mathbf{0}, \\
\Xi(\mathbf{q}, \dot{\mathbf{q}}, \boldsymbol{\lambda})=\mathbf{0},
\end{gathered}
$$

where the vector $\mathbf{q}$ contains the generalized coordinates of each fiber segment (positions and orientations) and $\boldsymbol{\lambda}$ is the vector of constraint forces ( $\mathbf{X}$ and $\left.\mathbf{F}^{\text {fric }}\right)$ in the suspension. If the segment orientations are represented by Euler parameters [Wittenberg (1977)], there are $7 N_{\text {fib }} N_{\text {seg }}$ equations of motion to be solved. The inextensibility constraint [Eq. (7)] represented by the vector $\boldsymbol{\Psi}$ is made up of $3 N_{\mathrm{fib}}\left(N_{\mathrm{seg}}-1\right)$ constraint equations that depend on only the positions and orientations. The $3 N_{C}$ friction constraint equations [Eq. (8)] are contained in $\Xi$ where $N_{C}$ is the total number of contacts in the system.

Simulations are performed by randomly placing fibers in their shape at equilibrium into a simulation cell of size $(\zeta L)^{3}$ as illustrated in Fig. 2, where $\zeta$ is the cell size scaling factor $(\zeta=1.5-4)$. A linear shear field is imposed and periodic boundary conditions are applied with the Lees-Edwards modification for shearing systems [Allen and Tildesley (1991)] to simulate an infinite suspension. Fiber motion is obtained by numerical solution of the system of DAEs in Eq. (10). An approximate solution method was developed to solve this system, details of which are found in the work of Schmid et al. (2000) and of Switzer (2002).

The particle positions and orientations are postprocessed in order to calculate the stress and other suspension properties. Using slender body theory, the extra particle stress [Eq. (3)] may be simplified to the form [Batchelor (1970a); Switzer (2002)]

$$
\begin{aligned}
\left\langle\boldsymbol{\sigma}^{p}\right\rangle= & \frac{4 \pi n \ell^{3} \eta_{0}}{3 \ln \left(2 r_{p}\right)}\left\langle\sum _ { i = 1 } ^ { N _ { \mathrm { seg } } } \left\{\mathbf{E}^{\infty} \cdot \mathbf{p}_{i} \mathbf{p}_{i}+\mathbf{p}_{i} \mathbf{p}_{i} \cdot \mathbf{E}^{\infty}-\mathbf{p}_{i} \mathbf{p}_{i} \mathbf{p}_{i} \mathbf{p}_{i}: \mathbf{E}^{\infty}-\left(\mathbf{p}_{i} \dot{\mathbf{p}}_{i}+\dot{\mathbf{p}}_{i} \mathbf{p}_{i}\right)\right.\right. \\
& \left.\left.+\frac{3}{\ell^{2}}\left(\left[\boldsymbol{\delta}-\frac{1}{2} \mathbf{p}_{i} \mathbf{p}_{i}\right] \cdot\left(\mathbf{U}^{\infty}-\dot{\mathbf{r}}_{i}\right) \mathbf{r}_{i}+\mathbf{r}_{i}\left[\boldsymbol{\delta}-\frac{1}{2} \mathbf{p}_{i} \mathbf{p}_{i}\right] \cdot\left(\mathbf{U}^{\infty}-\dot{\mathbf{r}}_{i}\right)\right)\right\}\right)+\Upsilon \boldsymbol{\delta},
\end{aligned}
$$

where $Y$ represents an isotropic contribution of no interest. Suspensions were sheared for at least 500 strain units after reaching steady state. The particle stress for particular runs was averaged over all configurations that were saved after steady state was reached. Most data reported in Sec. III are for single runs. Some runs were replicated with different initial conditions; uncertainties estimated from the replicates are indicated by error bars in the figures. 


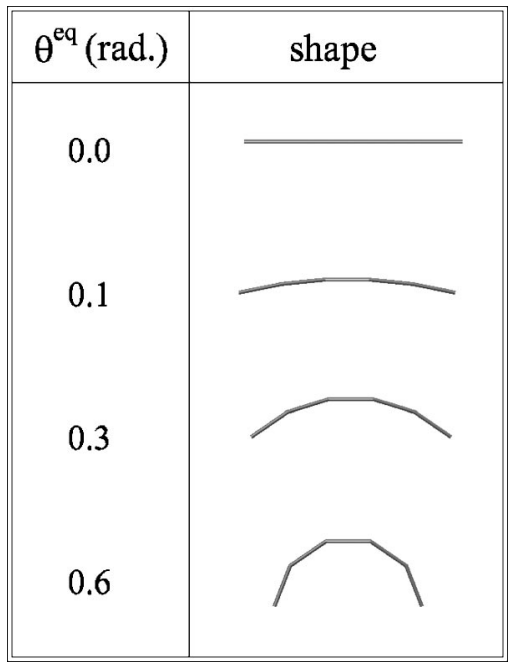

FIG. 3. Examples of U-shaped fibers ( $\left.\phi^{\mathrm{eq}}=0, r_{p}=75\right)$ for various values of $\theta^{\mathrm{eq}}$.

\section{RESULTS AND DISCUSSION}

\section{A. Effects of fiber shape and friction}

Here we investigate the effects of fiber shape and static friction on the shear viscosity of fiber suspensions. Simple shear flow was simulated for suspensions of U-shaped fibers $\left(r_{p}=75, S^{\text {eff }}=0.05\right)$ at various concentrations and for several different equilibrium bending angles and coefficients of friction. U-shaped fibers with different equilibrium bending angles are illustrated in Fig. 3.

The specific viscosity $\eta_{\mathrm{sp}} \equiv \eta / \eta_{0}-1\left(\eta \equiv \sigma_{z x} / \dot{\gamma}\right.$ is the suspension viscosity) is plotted as a function of the concentration $\left(n L^{3}\right)$ in Figs. 4(a) and 4(b) for suspensions of U-shaped fibers. Results are presented for simulations of flexible fibers $\left(S^{\text {eff }}=0.05, r_{p}\right.$ $\left.=75, N_{\text {seg }}=5\right)$ with $\theta^{\mathrm{eq}}=0,0.1$, and 0.3 , as well as for straight, rigid fibers $\left(r_{p}\right.$ $\left.=75, N_{\text {seg }}=1\right)$. Results for simulations without friction $\left(\mu^{\text {stat }}=0\right)$ are represented by open symbols, and results for simulations with friction $\left(\mu^{\text {stat }}=20\right)$ are represented by closed symbols. Figure 4(b) illustrates data over wider ranges of concentrations and specific viscosities than those presented in Fig. 4(a). The data points indicate individual simulation runs in which the average value is computed from at least 500 steady state configurations. Uncertainties (95\% confidence interval) estimated from replicate simulations with different initial configurations are indicated by error bars.

Consider first the effect of fiber flexibility in the absence of friction in these simulations, illustrated by the results for suspensions of rigid, straight fibers (open circles) and the results for suspensions of flexible, straight fibers (open squares) in Fig. 4(a). For both systems, $\eta_{\mathrm{sp}}$ is small, and increases roughly linearly with the concentration in agreement with previous simulations at relatively small concentrations [Sundararajakumar and Koch (1997)]. The linear dependence of the viscosity on concentration is consistent with the predictions of slender body theory for dilute fiber suspensions. For an ambient flow field $\mathbf{U}^{\infty}=(\dot{\gamma} z, 0,0)$, the slender body theory prediction for the specific viscosity in a dilute suspension of rigid rods is [Batchelor (1970a)]

$$
\eta_{\mathrm{sp}}^{\mathrm{sb}}=n L^{3} \frac{\pi}{6 \ln \left(2 r_{p}\right)}\left\langle p_{x}^{2} p_{z}^{2}\right\rangle-1
$$



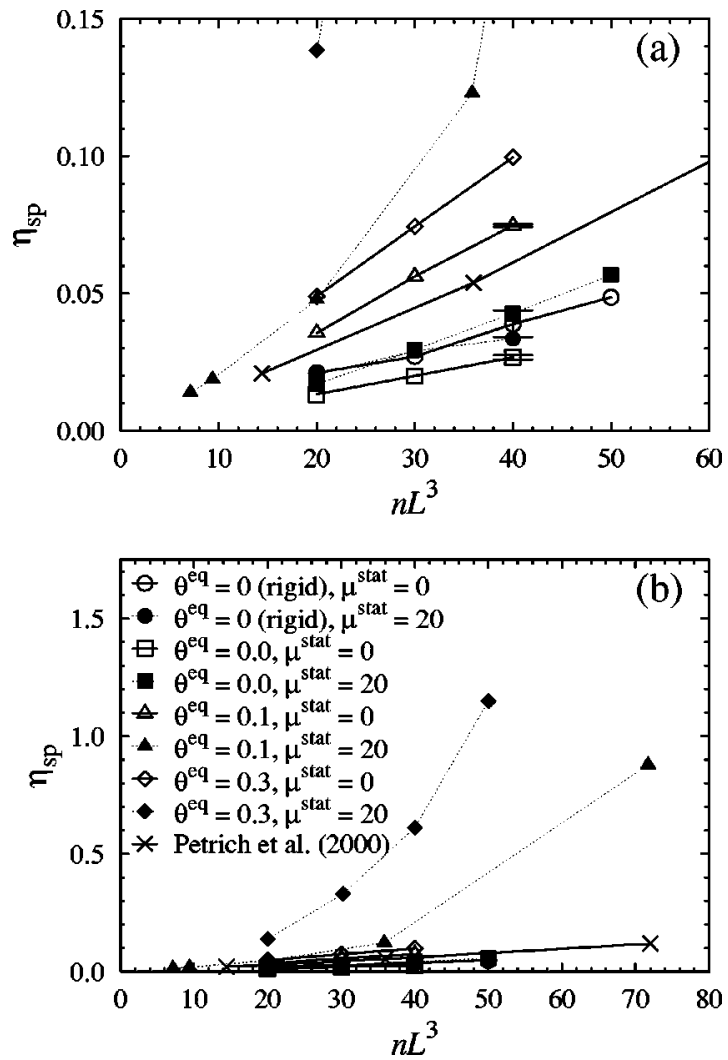

FIG. 4. Steady state specific viscosity as a function of the concentration for suspensions with fibers of different shape and coefficients of friction.

For suspensions of noninteracting particles, the orientation distribution is approximately constant which suggests $\eta_{\mathrm{sp}}^{\mathrm{sb}} \propto n L^{3}$. Petrich et al. (2000) also observed this type of behavior in experiments of very stiff and straight glass fibers $\left(S^{\text {eff }} \approx 1\right)$ with an aspect ratio $r_{p}=72$ at approximately the same concentration range as our simulations (illustrated in Fig. 4).

The differences between the results for suspensions of straight, rigid fibers and suspensions of straight, flexible fibers are very small, approximately equal to the uncertainties. This is not unexpected, since the flexible fibers in this case are actually quite stiff. For these simulations, the average deviation of the bending angles from the equilibrium value is $\left\langle\left|\theta_{i}-\theta^{\mathrm{eq}}\right|\right\rangle=6 \times 10^{-4}$. Indeed, the degree of deformation for all the runs depicted in Fig. 4 is quite small, and thus the results appear to represent the behavior of stiff fibers $\left(\left\langle\left|\theta_{i}-\theta^{\mathrm{eq}}\right|\right\rangle=4.6 \times 10^{-3}\right.$ for $\theta^{\mathrm{eq}}=0.1$ and $\left\langle\left|\theta_{i}-\theta^{\mathrm{eq}}\right|\right\rangle=1.6 \times 10^{-2}$ for $\left.\theta^{\mathrm{eq}}=0.3\right)$. The effects of flexibility for suspensions of more flexible fibers are discussed in Sec. III B.

Next we consider the effect of fiber shape in the absence of friction, illustrated by the results for straight, rigid and flexible fibers (open circles and squares, respectively), and U-shaped, flexible fibers ( $\theta^{\mathrm{eq}}=0.1$, open triangles) in Fig. 4(a). The specific viscosities of the suspensions of U-shaped fibers are significantly larger than those of the suspensions of straight fibers. At $n L^{3}=40, \eta_{\text {sp }}$ for the U-shaped fibers is approximately twice 


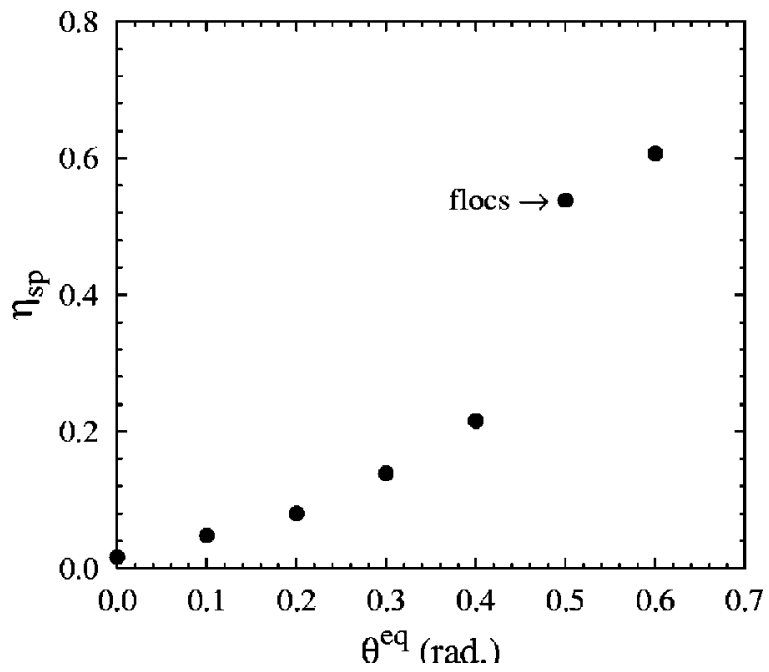

FIG. 5. Specific viscosity as a function of the equilibrium shape of U-shaped fibers after shearing for $\gamma$ $=1500 ;\left(N_{\mathrm{seg}}, r_{p}, n L^{3}, S^{\text {eff }}, \phi^{\text {eq }}, \mu^{\text {stat }}\right)=(5,75,20,0.05,0,20)$.

as large as that for the straight fibers. We note that for $\theta^{\mathrm{eq}}=0.1$, the fiber shape is nearly straight (see Fig. 3). Thus a small amount of curvature can have a significant effect on the suspension viscosity.

The results presented in Fig. 4 also illustrate the impact of interfiber friction on the shear viscosity. For suspensions of straight, rigid fibers, adding static friction ( $\mu^{\text {stat }}$ $=20$ ) does not alter the shear viscosity. For suspensions of straight, flexible fibers, adding static friction produces a small increase in the specific viscosity. The effect of static friction is much more pronounced for the U-shaped fibers. For $n L^{3} \gtrsim 30$, adding static friction more than doubles the specific viscosity. In addition, the concentration dependence changes from a linear dependence in the absence of friction to a higher-order dependence with $\mu^{\text {stat }}=20$.

The viscosity of fiber suspensions is thus significantly influenced by both the fiber shape and static friction. Furthermore, there is also an additional synergistic effect of these features, because friction influences the viscosity of suspensions of U-shaped fibers more than it influences the viscosity of suspensions of straight fibers. The effects of fiber shape and friction are explored in more detail below.

The effect of shape on the specific viscosity of suspensions of U-shaped fibers is further illustrated in Fig. 5, in which all other suspension properties are held constant $\left[\left(N_{\text {seg }}, r_{p}, n L^{3}, S^{\text {eff }}, \phi^{\text {eq }}, \mu^{\text {stat }}\right)(5,75,20,0.05,0,20)\right]$. The specific viscosity gradually increases as the fiber curvature increases ( $\theta^{\text {eq }}$ increases). At a certain curvature, $\theta^{\text {eq }}$ $=0.5$ for the conditions listed above, the specific viscosity undergoes a large jump in value. This corresponds to the formation of fiber flocs in the suspension. Further increases in the equilibrium bending angle also result in a flocculated suspension and a gradual increase in $\eta_{\text {sp }}$. The effect of flocculation on rheological properties is discussed further in Sec. III D.

Deviation of fiber shape from perfectly straight can influence the stress in the suspension in several ways. Consider first dilute suspensions in which the fiber rotation dynamics are similar to those of isolated fibers. U-shaped fibers have a smaller period of rotation than straight fibers of the same aspect ratio, and thus spend a larger fraction of time out 


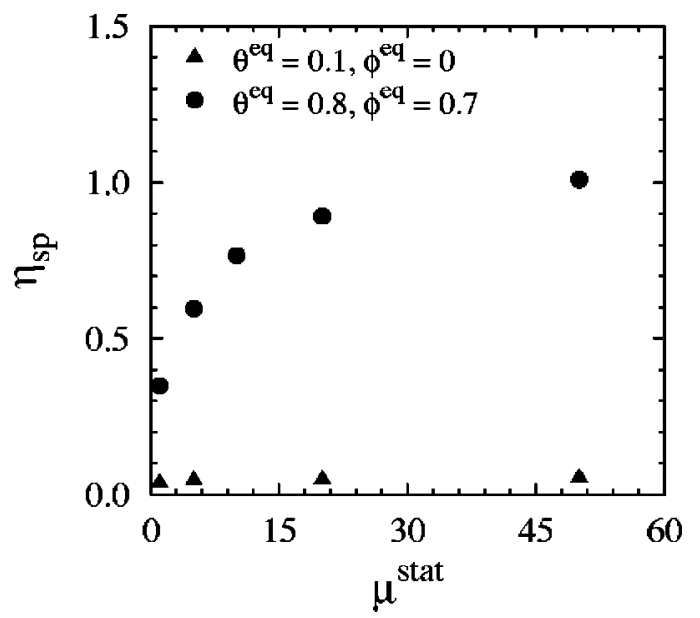

FIG. 6. Specific viscosity as a function of the static coefficient of friction for suspensions of fibers that are $U$ shaped $\left(\theta^{\mathrm{eq}}=0.1, \phi^{\mathrm{eq}}=0\right)$ and helical $\left(\theta^{\mathrm{eq}}=0.8, \phi^{\mathrm{eq}}=0.7\right)$ after shearing for $\gamma=1500$ with all other parameters constant; $\left(N_{\mathrm{seg}}, n L^{3}, r_{p}\right)=(5,20,75)$.

of the plane of shear. In addition, for U-shaped fibers, some fiber segment orientation vectors $\mathbf{p}_{i}$ will always have nonzero components normal to the plane of shear (except in the rare situation in which the plane of the "U" lies within a shearing plane), in contrast to straight fibers that spend most of the time nearly aligned with the flow and contribute little to the suspension viscosity. These features directly affect the hydrodynamic contribution to the stress represented by the integral term in Eq. (3). Fiber shape can also influence the nonhydrodynamic contribution to stress [represented by the terms that contain products of $\mathbf{F}^{H}$ and $\mathbf{r}$ in Eq. (3)] by impacting the frequency of contact between fibers. Interfiber contacts result in nonzero hydrodynamic forces $\mathbf{F}^{H}$, and thus more frequent contacts are expected to increase the time-averaged stress. The contacts also affect the fiber segment orientation distribution, and indirectly influence the hydrodynamic contribution to the stress. Fiber curvature indeed leads to an increase in the number of contacts. For the simulation runs depicted in Fig. 5, the average number of contacts per fiber at steady state $\left\langle n_{c}\right\rangle_{\mathrm{ss}}$ increases from 0.03 for $\theta^{\mathrm{eq}}=0$ to 1.12 for $\theta^{\mathrm{eq}}=0.3$.

The increase in the number of contacts per fiber as the fiber curvature increases arises from two features. The shorter rotation period of U-shaped fibers compared to that for straight fibers of the same aspect ratio leads to more opportunities for fiber contacts. U-shaped fibers also tend to sweep out a larger volume as they rotate, further increasing the probability of contact with other fibers. These effects of shape on the number of contacts per fiber should hold for any type of deviation from straight shape (e.g., U shaped, helical, etc.).

Figure 6 demonstrates the effect of changing $\mu^{\text {stat }}$ on the specific viscosity of fiber suspensions with two different equilibrium shapes [U shaped: $\left(\theta^{\mathrm{eq}}, \phi^{\mathrm{eq}}\right)=(0.1,0)$; and helical: $\left.\quad\left(\theta^{\mathrm{eq}}, \phi^{\mathrm{eq}}\right)=(0.8,0.7)\right]$ with all other parameters held constant $\left[\left(N_{\text {seg }}, n L^{3}, r_{p}, S^{\text {eff }}\right)=(5,20,75,0.05)\right]$. The specific viscosity of suspensions of U-shaped fibers first increases as $\mu^{\text {stat }}$ increases, and then approaches a constant. The helical fiber suspensions show a more substantial change in specific viscosity as $\mu^{\text {stat }}$ increases. This may be attributed in part to an increase in the number of contacts. For a value of $\mu^{\text {stat }}=5$, the number of contacts per fiber at steady state for the U-shaped fibers is $\left\langle n_{c}\right\rangle_{\mathrm{ss}}=0.08$, while for the helical fibers $\left\langle n_{c}\right\rangle_{\mathrm{ss}}=2.3$. The helical fibers also floccu- 

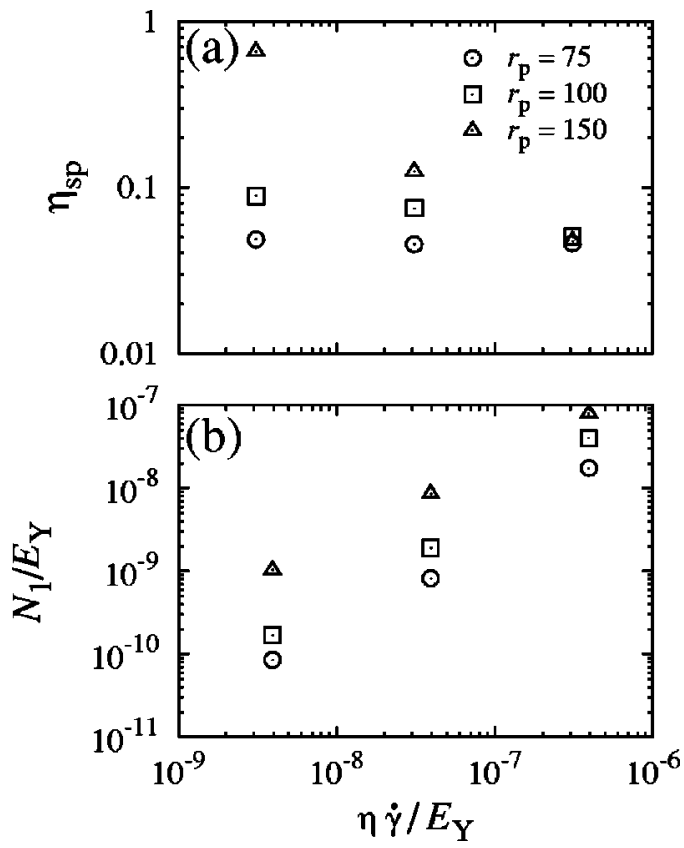

FIG. 7. (a) Specific viscosity and (b) normalized first normal stress difference $N_{1} / E_{Y}$ as a function of the dimensionless shear rate $\eta_{0} \dot{\gamma} / E_{Y}$ for fibers of various aspect ratios after shearing for $\gamma=1500$; $\left(N_{\text {seg }}, r_{p}, \Phi, \theta^{\text {eq }}, \phi^{\text {eq }}, \mu^{\text {stat }}\right)=(5,75,0.003,0.1,0,20)$.

late for $\mu^{\text {stat }} \geqslant 5$; flocculated suspensions show different rheological behavior than homogeneous suspensions as will be described in Sec. III D.

\section{B. Effects of aspect ratio and stiffness}

Suspensions of flexible, U-shaped fibers in simple shear flow were simulated at a fixed volume fraction, $\Phi=0.003$, with three different aspect ratios, $r_{p}=75,100$, and 150 $\left(n L^{3}=21.5,38.2\right.$, and 85.9, respectively), and for various values of the dimensionless stiffness $S^{\text {eff }}=E_{Y} I / \eta_{0} \dot{\gamma} L^{4}$. All other parameter values were fixed at $\left(N_{\text {seg }}, \theta^{\text {eq }}, \phi^{\text {eq }}, \mu^{\text {stat }}\right)=(5,0.1,0,20)$. The steady state specific viscosity is plotted as a function of the dimensionless shear rate $\eta_{0} \dot{\gamma} / E_{Y}$ in Fig. 7(a) for the various values of $r_{p}$. All suspensions show shear thinning behavior over the range of dimensionless shear rates investigated. As the aspect ratio increases, the shear thinning behavior becomes more pronounced. At large shear rates, or for sufficiently flexible fibers, the specific viscosities exhibit a much weaker dependence on the aspect ratio than that observed at low shear rates. Figure 7(b) shows the difference in first normal stress normalized by the fiber's Young's modulus $N_{1} / E_{Y}$ as a function of $\eta_{0} \dot{\gamma} / E_{Y}$ for the same fiber aspect ratios discussed above. The first normal stress difference also increases with increasing aspect ratio, and the increase is more noticeable at lower shear rates. The dependence of the rheological properties on the fiber aspect ratio and shear rate observed in the simulations is consistent with the experimental results of Goto et al. (1986) for nylon fibers in glycerin and of Kitano and Kataoka (1981) for vinylon fibers in silicone oil.

The simulation results presented in Fig. 7(a) suggest that increasing the fiber flexibility (decreasing $E_{Y}$ ) will lead to smaller suspension viscosity. This apparently contradicts the experimental results of Goto et al. (1986) which demonstrate that nylon fiber suspensions 


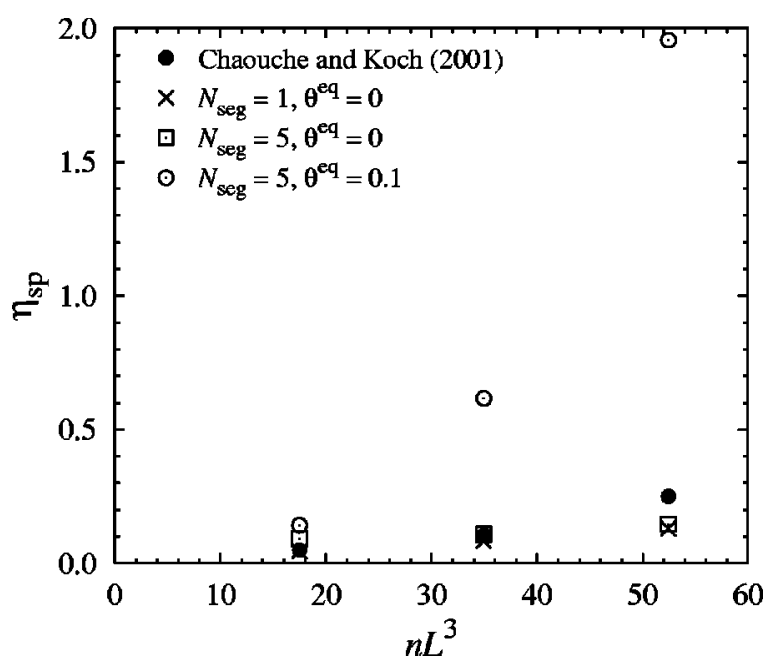

FIG. 8. Specific viscosity as a function of the concentration for shorter fiber suspensions $\left(r_{p}=35\right)$, using rigid fibers $\left[\left(N_{\mathrm{seg}}, S^{\text {eff }}, \mu^{\text {stat }}\right)=(1, \infty, 20)\right]$, straight flexible fibers $\left[\left(N_{\text {seg }}, S^{\text {eff }}, \theta^{\text {eq }}, \phi^{\text {eq }}, \mu^{\text {stat }}\right)\right.$ $=(5,0.7,0,0,20)]$, and slightly deformed flexible fibers $\left[\left(N_{\mathrm{seg}}, S^{\mathrm{eff}}, \theta^{\mathrm{eq}}, \phi^{\mathrm{eq}}, \mu^{\text {stat }}\right)=(5,0.7,0.1,0,20)\right]$ compared with the experimental data of Chaouche and Koch (2001).

$\left(E_{Y} \approx 2 \mathrm{GPa}\right)$ have larger viscosities than glass fiber suspensions $\left(E_{Y} \approx 75 \mathrm{GPa}\right)$ at the same aspect ratio $\left(r_{p}=300\right)$ and concentration $(\Phi=0.005)$. This discrepancy may be explained by examining the equilibrium shapes of the nylon and glass fibers depicted by Goto et al. The glass fibers appear to be nearly straight at equilibrium, whereas the nylon fibers have permanent deformation at equilibrium. As described in Sec. III A, the differences in the rheological properties of the glass and nylon fibers observed by Goto et al. may be attributed to differences in fiber shape as well as differences in fiber stiffness.

We also performed simulations for suspensions of fibers with aspect ratio $r_{p}=35$ in order to compare with the experimental results of Chaouche and Koch (2001) for nylon fibers in silicone oil $\left(r_{p}=36, \eta_{0}=12.2 \mathrm{Pas}\right.$ ). For this system at a shear rate of $\dot{\gamma}$ $=9.8 \mathrm{~s}^{-1}$, the effective stiffness is $S^{\text {eff }} \approx 0.8$. Under these conditions, the suspensions appeared to be essentially Newtonian. Simulation results for the steady state specific viscosity as a function of the concentration for suspensions of fibers of various shape are compared with the experimental results of Chaouche and Koch (2001) in Fig. 8. The results for suspensions of rigid rods $\left[\left(N_{\text {seg }}, S^{\text {eff }}, \theta^{\text {eq }}, \phi^{\text {eq }}, \mu^{\text {stat }}\right)=(1, \infty, 0,0,20)\right]$ agree reasonably well with the experimental measurements, with deviations becoming more apparent as the concentration increases. As described in Sec. III A, friction does not significantly affect the results for straight fibers in the concentration range reported. If the fibers are made flexible and inherently straight with a dimensionless stiffness similar to that of the experimental system $\left[\left(N_{\text {seg }}, S^{\text {eff }}, \theta^{\text {eq }}, \phi^{\text {eq }}, \mu^{\text {stat }}\right)=(5,0.7,0,0,20)\right]$, there is only a slight improvement in the prediction of the specific viscosity at large concentrations. Simulations with slightly U-shaped, flexible fibers $\left[\left(N_{\mathrm{seg}}, S^{\mathrm{eff}}, \theta^{\mathrm{eq}}, \phi^{\mathrm{eq}}, \mu^{\text {stat }}\right)\right.$ $=(5,0.7,0.1,0,20)]$ overpredict $\eta_{\mathrm{sp}}$ by nearly an order of magnitude. These results suggest that the small discrepancy between the experimental results and the predictions for suspensions of straight fibers may be accounted for by a very small deviation of the shape (from perfectly straight) of the fibers employed in the experiments. 


\section{Yield stress}

Fibers in suspension can form networks if the concentration is sufficiently large that each fiber experiences multiple contacts with other fibers. The networks exhibit mechanical strength and viscoelastic behavior [Wahren (1964); Thalén and Wahren (1964)]. The network strength is controlled by the cohesive nature of the contact points. Kerekes et al. (1985) proposed that the cohesive force that imparts strength to the network is caused by friction generated by normal forces at points of contact between elastically bent fibers. Bennington et al. (1990) derived an expression for the yield stress of a suspension of elastically interlocked fibers,

$$
\sigma_{0}=\alpha \Phi^{3}
$$

where $\sigma_{0}$ is the yield stress and the coefficient $\alpha$ is a function of the fiber's Young's modulus and aspect ratio. Bennington et al. measured the yield stress for a variety of suspensions of wood and synthetic fibers, and found that the yield stress scaled with the volume fraction as $\sigma_{0} \sim \Phi^{\beta}$ where $\beta$ ranged from 2.5 to 3.5 , in reasonable agreement with their model.

We performed simulations in simple shear flow to determine the yield stress of fiber suspensions at various volume fractions with $\left(N_{\mathrm{seg}}, r_{p}, \theta^{\mathrm{eq}}, \phi^{\mathrm{eq}}, \mu^{\text {stat }}\right)$ $=(5,75,0.1,0,20)$. The stiffness was varied to obtain the dimensionless shear stress $\left(\sigma_{z x} L^{4} / E_{Y} I\right)$ as a function of the dimensionless shear rate $\left(1 / S^{\mathrm{eff}} \propto \dot{\gamma}\right)$, which is plotted for different volume fractions in Fig. 9(a). The data exhibit roughly Bingham-like behavior, with the shear stress varying linearly with the shear rate and a nonzero intercept. The apparent dimensionless yield stress at each volume fraction was determined by fitting the data to a straight line and equating the intercept at $1 / S^{\mathrm{eff}}=0$ to the dimensionless (dynamic) yield stress. The yield stresses obtained in this manner are plotted as a function of volume fraction in Fig. 9(b). The simulated yield stress scales with the volume fraction as $\sigma_{0} \sim \Phi^{2.75 \pm 0.8}$, and thus the exponent is in the same range as the values obtained experimentally by Bennington et al. (1990).

The predicted value of $\alpha$ does not agree as well with that obtained experimentally. Using the parameter values for nylon fibers $r_{p} \approx 76, E_{Y} I \approx 3.5 \times 10^{-10} \mathrm{~N} \mathrm{~m}^{2}$, and $L$ $\approx 3.45 \mathrm{~mm}$ [Bennington et al. (1990)], the simulations predict a value of $\alpha \approx 6$ $\times 10^{6} \mathrm{~N} / \mathrm{m}^{2}$, whereas Bennington et al. measured $\alpha \approx 10^{5} \mathrm{~N} / \mathrm{m}^{2}$. The discrepancy between the simulated and experimentally measured values of $\alpha$ may be explained by a variety of effects. The fiber shapes and values of the coefficients of friction employed in the simulations certainly differ from those of the experimental systems and, as discussed in Sec. III A, we expect the stress to depend fairly sensitively on these parameter values. Unfortunately, the fiber shapes and coefficients of friction for the systems employed in the experiments were not reported.

The network model of Bennington et al. (1990) suggests that the yield stress should vary linearly with the fiber's Young's modulus. The same conclusion is obtained for the simulations reported here, since the yield stress scaled by the Young's modulus is obtained by extrapolating the dimensionless shear stress to $1 / S^{\text {eff }} \rightarrow 0$. The dimensionless yield stress is thus independent of the Young's modulus, and the dimensional yield stress varies linearly with the Young's modulus. Bennington et al., however, did not observe this scaling in their experiments. This discrepancy may also be related to the fiber properties. Bennington et al. obtained results for different fiber Young's moduli by employing different types of wood and synthetic fibers. Inspection of the fiber images presented in their paper reveals that the fiber shapes varied, sometimes quite significantly, from one system to another. Thus the variation of yield stress from one system to another may be 

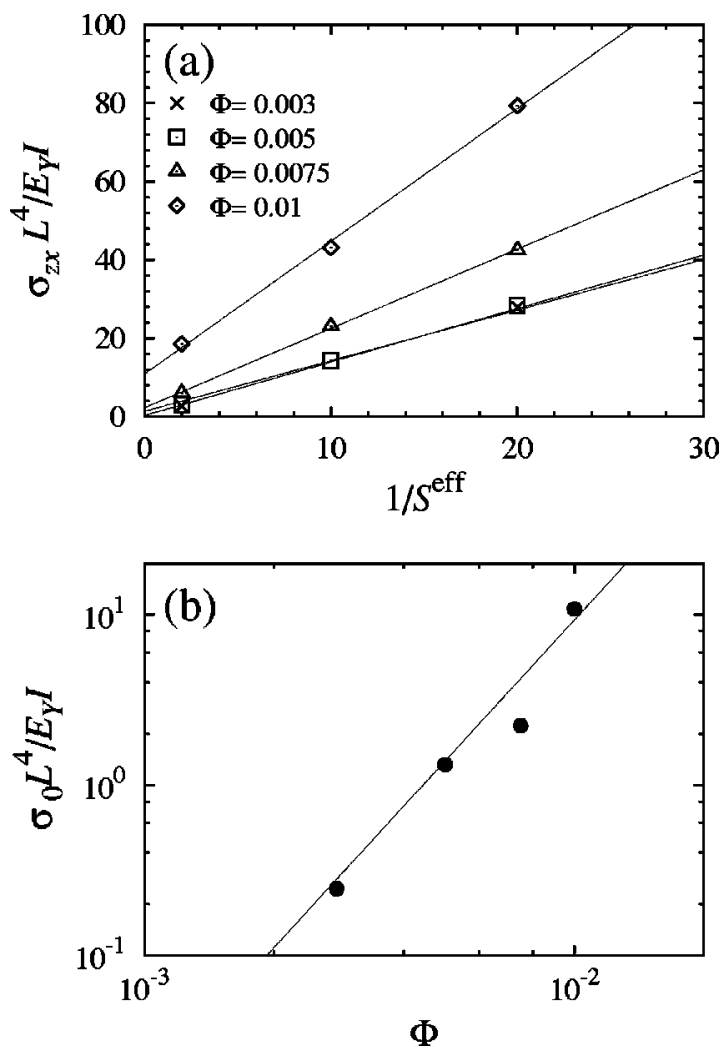

FIG. 9. (a) Shear stress $\left(\sigma_{x z}\right)$ as a function of the shear rate for simulations of fiber suspensions at different volume fractions, and (b) the extrapolated apparent yield stress $\left(\sigma_{0}\right)$ from (a) as a function of the volume fraction; $\left(N_{\mathrm{seg}}, r_{p}, \theta^{\mathrm{eq}}, \phi^{\mathrm{eq}}, \mu^{\text {stat }}\right)=(5,75,0.1,0,20)$.

due to differences in fiber shape as well as to differences in Young's moduli, and thus the predicted scaling with stiffness alone is not expected to hold.

\section{Rheology in flocculated suspensions}

Under the appropriate conditions, fiber networks subjected to shear flow develop into a heterogeneous or flocculated state. Flocculation can occur when the fibers are sufficiently stiff and interact with a sufficiently large coefficient of friction, even in the absence of attractive interfiber forces [Schmid et al. (2000), Switzer (2002)]. Fibers within flocs are caught in elastically strained configurations due to fiber bending and friction forces at the contact points. A flocculated state in a simulated fiber suspension can be characterized by the pair distribution function, $g(r)$, where $r$ is the separation between fiber centers of mass. Pair distribution functions for two fiber suspensions after shearing to steady state (strain $\gamma=1500$ ) are plotted in Fig. 10(a). The parameter values for the two simulations are the same $\left[\left(N_{\mathrm{seg}}, r_{p}, n L^{3}, S^{\mathrm{eff}}, \theta^{\mathrm{eq}}, \phi^{\mathrm{eq}}\right)=(5,75,15,0.05,0.8,0.7)\right]$, except for the values of the coefficient of friction $\left(\mu^{\text {stat }}=1\right.$ and 20). For $\mu^{\text {stat }}=1$, fibers have an equal probability of having their centers of mass at any separation. This resulting suspension structure is homogeneous, as illustrated in Fig. 10(c). For sufficiently large values of $\mu^{\text {stat }}$, the suspension flocculates [Fig. 10(b)] and there is a high probability of finding the fiber centers of mass at small separations. 


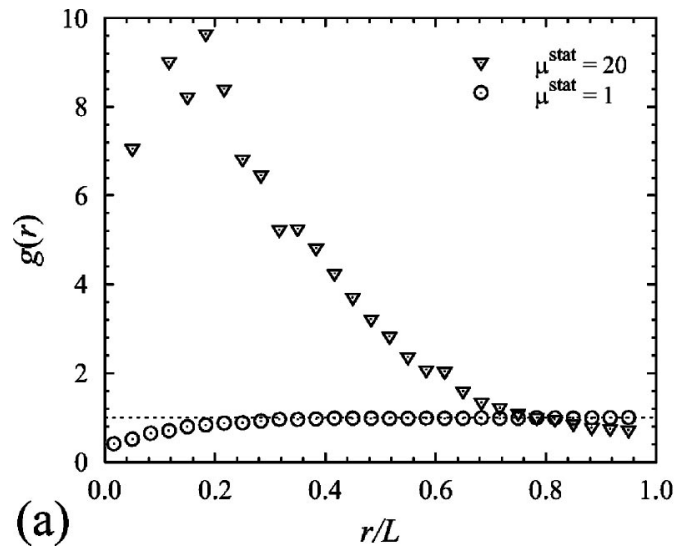

(b)

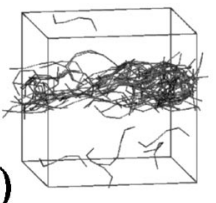

(c)

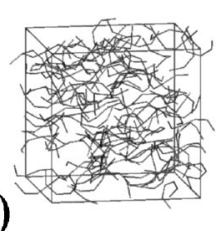

FIG. 10. Pair distribution function for the fiber centers of mass for a flocculated suspension $\left(\mu^{\text {stat }}=20\right)$ and a homogeneous suspension $\left(\mu^{\text {stat }}=1\right)$ after shearing for $\gamma=1500 ; \quad\left(N_{\text {seg }}, r_{p}, n L^{3}, S^{\text {eff }}, \theta^{\text {eq }}, \phi^{\text {eq }}\right)$ $=(5,75,15,0.05,0.1,0)$.

Flocculation changes the rheological properties of a suspension. Figure 11 shows the specific viscosity as a function of $1 / S^{\text {eff }}(\propto \dot{\gamma})$ for fiber suspensions with the same concentration and aspect ratio $\left(n L^{3}=15\right.$ and $\left.r_{p}=75\right)$, but with different shapes and coefficients of friction. Results labeled (A) are for simulations with helical fibers $\left[\left(\theta^{\mathrm{eq}}, \phi^{\mathrm{eq}}\right)=(0.8,0.7)\right]$ with $\mu^{\text {stat }}=20$, results labeled (B) are for simulations with the same helical fiber shape and $\mu^{\text {stat }}=1$, and results labeled (C) are for runs with U-shaped fibers $\left[\left(\theta^{\mathrm{eq}}, \phi^{\mathrm{eq}}\right)=(0.1,0)\right]$ and $\mu^{\text {stat }}=20$. Systems (B) and (C) remain homogeneous for all values of $S^{\text {eff }}$. The specific viscosity exhibits a low shear rate plateau, with shear thinning behavior as $1 / S^{\text {eff }}$ increases. The value of $\eta_{\mathrm{sp}}$ at small values of $1 / S^{\mathrm{eff}}$ is larger for system (B) than for system (C), because the fiber equilibrium shapes in system (B) deviate more from perfectly straight. Thus, fiber segments in system (B) are never all

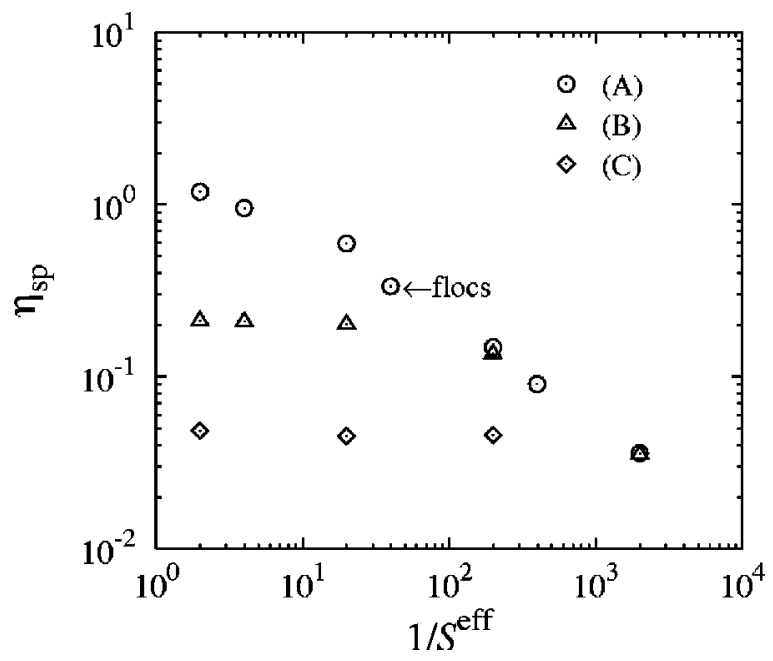

FIG. 11. Specific viscosity vs the reciprocal of the stiffness for suspensions of equivalent concentration $\left(n L^{3}=15\right)$ and aspect ratio $\left(r_{p}=75\right)$ in which (A) flocculates and (B) and (C) remain homogeneous: (A) $\left(\theta^{\mathrm{eq}}, \phi^{\mathrm{eq}}, \mu^{\text {stat }}\right)=(0.8,0.7,20) ;(\mathrm{B})\left(\theta^{\mathrm{eq}}, \phi^{\mathrm{eq}}, \mu^{\text {stat }}\right)=(0.8,0.7,1) ;(\mathrm{C})\left(\theta^{\mathrm{eq}}, \phi^{\mathrm{eq}}, \mu^{\text {stat }}\right)=(0.1,0,20)$. 
aligned with the flow direction, which leads to a larger hydrodynamic contribution to stress compared to the slightly deformed fibers in system (C) which can approximately align with the direction of flow. The helical fibers also experience more interfiber contacts, further increasing the stress.

System (A) remains homogeneous at large values of $1 / S^{\text {eff }}$ (high shear rates), but for $1 / S^{\mathrm{eff}} \lesssim 400$ the suspension flocculates at steady state [following Schmid et al. (2000), we define a suspension as flocculated when $g(r=0.01 L) \geqslant 3]$. Shear thinning behavior is observed over the entire range of $1 / S^{\mathrm{eff}}$. The specific viscosity is also much larger in the flocculated state at small values of $1 / S^{\text {eff }}$ because there are many frictional contacts that give the network mechanical strength. Chen et al. (2002) observed large jumps in the shear stress for wood and nylon fiber suspensions when they flocculated at small shear rates. The simulation results depicted in Fig. 11 do not exhibit large jumps in shear stress over the range of dimensionless shear rates investigated, but may do so at smaller shear rates.

At large values of $1 / S^{\text {eff }}$, all of the systems described in Fig. 11 approach the same value for $\eta_{\mathrm{sp}}$, where the suspension structures are homogeneous. This is consistent with the results of Chen et al. (2002) in which they observed that suspensions dispersed from a flocculated state as the shear rate increased. Goto et al. (1986) also reported that fibers of various Young's moduli and shape tended to approach the same viscosity at high shear rates (for equivalent concentrations), where the suspensions behaved essentially like Newtonian fluids. This is consistent with the simulation results reported here, and corresponds to a limiting state where viscous forces dominate over fiber elasticity and interfiber forces.

\section{CONCLUSIONS}

We have employed particle-level simulations to probe the effects of various features of flexible fibers and their interactions on the rheology of fiber suspensions in simple shear flow. The fibers are modeled as linked, rigid spherocylinders connected by ball and socket joints. The fiber segments are acted upon by short-range repulsive forces and interfiber static friction, but hydrodynamic interactions are ignored. Since we were primarily interested in situations where there are significant numbers of interfiber contacts, hydrodynamic interactions are likely of secondary importance [Sundararajakumar and Koch (1997); Harlen et al. (1999)]. Comparisons with previous simulations and experiments show reasonable agreement.

The viscosity of fiber suspensions is influenced strongly by the particle shape and interfiber friction. Relatively small deviations in particle shape from perfectly straight can have $O(1)$ effects on the specific viscosity. Interfiber friction impacts the viscosity more for suspensions of nonstraight fibers than for suspensions of straight fibers. These results suggest that these features should be quantified in experimental studies.

Fiber flexibility results in shear thinning behavior. This is caused by competition between between hydrodynamic forces (which attempt to deform the fibers) and fiber elasticity (which attempts to retain the equilibrium fiber shape), characterized by a dimensionless effective stiffness, $S^{\text {eff }}=E_{Y} I / \eta_{0} \dot{\gamma} L^{4}$, where $E_{Y}$ is the Young's modulus of the fiber material, $I$ is the cross-sectional area moment, $\eta_{0}$ is the suspending fluid viscosity, $\dot{\gamma}$ is the shear rate, and $L$ is the fiber length; $1 / S^{\text {eff }}$ can be interpreted as a dimensionless shear rate. Shear thinning proceeds from a low shear rate plateau to a shape-independent high shear rate limit. The height of the low shear rate plateau depends on the fiber shape and the coefficient of friction. 
The behavior predicted at large shear rates is expected to be only qualitative, since deviations of the bending and twisting angles from their equilibrium values can become sufficiently large such that the Hookean model for bending and twisting torque in the hinges is suspect (e.g., in the most extreme case, $\left\langle\left|\theta-\theta^{\mathrm{eq}}\right|\right\rangle=0.086$ for the simulations with $\eta_{0} \dot{\gamma} / E_{Y}=3 \times 10^{-7}, r_{p}=150$ in Fig. 7). Furthermore, one would expect that fibers composed of relatively few rigid segments could not accurately represent the precise shapes exhibited by real fibers at large values of $1 / S^{\mathrm{eff}}$.

Suspensions that flocculate show shear thinning over wider ranges of shear rates. However, the high shear rate behavior of these systems is the same as that of suspensions that do not flocculate (at the same volume fraction). Results for the concentration dependence of the apparent yield stress agree reasonably well with experiments. The effects of fiber shape and friction make quantitative comparison with existing experimental data difficult.

\section{ACKNOWLEDGMENT}

This research was supported in part by the USDA NRI Competitive Grants Program (Award No. 2001-35103-09933).

\section{APPENDIX}

Here we investigate the error associated with neglecting hydrodynamic interactions between segments within a fiber by comparing the Jeffery orbit periods of straight, rigid cylinders in simple shear flow predicted from the model employed in this article with that predicted by Cox (1971).

Cox (1971) employed theoretical analysis and comparison with experiments to obtain the Jeffery orbit period of straight, rigid cylinders in shear flow,

$$
T=\frac{2 \pi}{\dot{\gamma}}\left(r_{e}+\frac{1}{r_{e}}\right),
$$

where the equivalent aspect ratio of the cylinder is given by

$$
r_{e}=1.24 \frac{r_{p}}{\sqrt{\ln r_{p}}} .
$$

These equations reproduced well experimental data reported by Anczurowski and Mason (1968).

Results for the orbit periods of straight, isolated fibers composed of linked spherocylinders predicted by the simulations in which hydrodynamic interactions between segments are ignored are compared with the results of Cox (1971) in Fig. 12, where the dimensionless period $T \dot{\gamma}$ is plotted as a function of $N_{\text {seg }}$ for different overall fiber aspect ratios $r_{p}$. The simulations were performed for sufficiently large values of $S^{\text {eff }}$ such that the orbit periods did not vary with further increases in $S^{\text {eff }}$. The solid lines in Fig. 12 represent the results given by Cox (1971).

The results presented in Fig. 12 illustrate fair agreement between the simulation model and theory, at least for the range of parameters explored. Most simulations reported in this article employ $r_{p}=75, N_{\text {seg }}=5$, for which the error in the orbit period is $0.5 \%$. The error increases with an increase in $r_{p}$, and reaches $11 \%$ for $r_{p}=150, N_{\text {seg }}=5$. This suggests that ignoring hydrodynamic interactions between segments does not have a significant impact on fiber dynamics. In principle, the equivalent aspect ratio of a seg- 


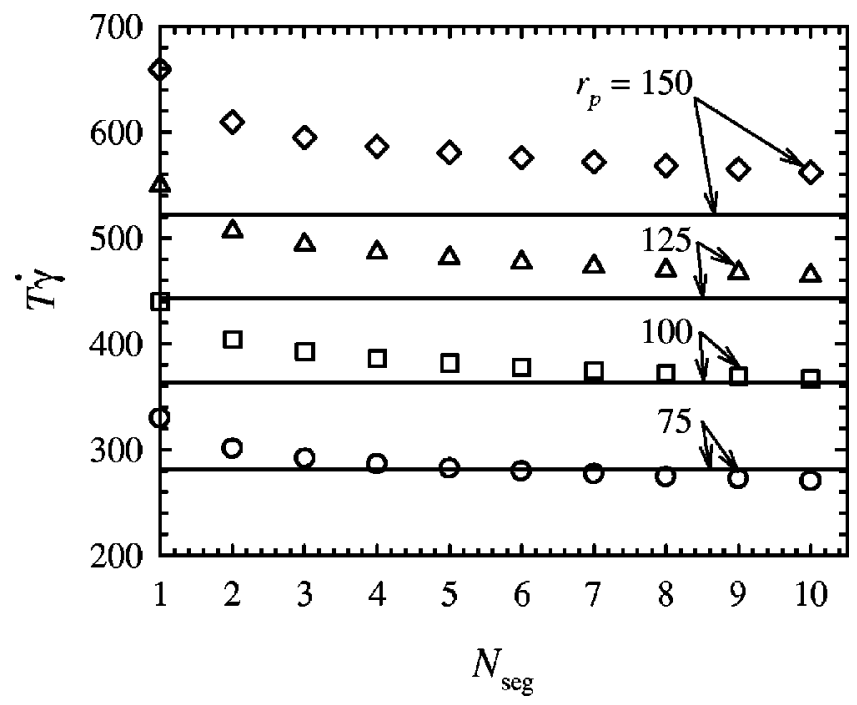

FIG. 12. Dimensionless orbit period as a function the number of segments for straight, rigid fibers in simple shear flow. The symbols represent predictions from the simulations in which hydrodynamic interactions between segments are ignored, and the lines represent results given by Cox (1971).

ment, $r_{e_{s}}$, could be adjusted to provide exact agreement, but this does not seem warranted given the other approximations employed (e.g., replacing a flexible fiber with linked, rigid segments).

\section{References}

Allen, M. P., and D. J. Tildesley, Computer Simulation of Liquids (Oxford Science, Oxford, 1991).

Anczurowski, E., and S. G. Mason, "Particle motions in sheared suspensions. XXIV. Rotation of rigid spheroids and cylinders," Trans. Soc. Rheol. 12, 209-215 (1968).

Batchelor, G. K., "Slender-body theory for particles of arbitrary cross-section in Stokes flow," J. Fluid Mech. 44, 419-440 (1970a).

Batchelor, G. K., "The stress system in a suspension of force-free particles," J. Fluid Mech. 41, 545-570 (1970b).

Bennington, C. P. J., R. J. Kerekes, and J. R. Grace, “The yield stress of fibre suspensions,” Can. J. Chem. Eng. 68, 748-757 (1990).

Bossis, G., and J. F. Brady, "Self-diffusion of Brownian particles in concentrated suspensions under shear," J. Chem. Phys. 9, 5437-5448 (1987).

Chaouche, M., and D. L. Koch, "Rheology of non-Brownian rigid fiber suspensions with adhesive contacts," J. Rheol. 45, 369-382 (2001).

Chen, B., D. Tatsumi, and T. Matsumoto, "Floc structure and flow properties of pulp fiber suspensions," J. Soc. Rheol., Jpn. 30, 19-25 (2002).

Claeys, I. L., and J. F. Brady, "Suspensions of prolate spheroids in Stokes flow. Part 1. Dynamics of a finite number of particles in an unbounded fluid," J. Fluid Mech. 251, 411-442 (1993a).

Claeys, I. L., and J. F. Brady, "Suspensions of prolate spheroids in Stokes flow. Part 2. Statistically homogeneous dispersions," J. Fluid Mech. 251, 443-477 (1993b).

Cox, R. G., "The motion of long slender bodies in a viscous fluid. Part 2. Shear flow," J. Fluid Mech. 45, 625-657 (1971).

Fan, X. J., N. Phan-Thien, and R. Zheng, "A direct simulation of fibre suspensions," J. Non-Newtonian Fluid Mech. 74, 113-135 (1998)

Goto, S., H. Nagazono, and H. Kato, "The flow behavior of fiber suspensions in Newtonian fluids and polymer solutions. I. Mechanical properties," Rheol. Acta 25, 119-129 (1986). 
Harlen, O. G., R. R. Sundararajakumar, and D. L. Koch, "Numerical simulations of a sphere settling through a supension of neutrally buoyant fibres," J. Fluid Mech. 388, 355-388 (1999).

Kerekes, R. J., R. M. Soszynski, and P. A. Tam Doo, “The flocculation of pulp fibres," in Papermaking Raw Materials, Transactions of the Eighth Fundamental Research Symposium, Oxford (Mechanical Engineering Publications Limited, London, 1985), pp. 265-310.

Kim, S., and S. J. Karrila, Microhydrodynamics: Principles and selected applications (ButterworthHeinemann, Stoneham, MA, 1991).

Kitano, T., and T. Kataoka, "The rheology of suspensions of vinylon fibers in polymer liquids. I. Suspensions in silicone oil,” Rheol. Acta 20, 390-402 (1981).

Mackaplow, M. B., and E. S. G. Shaqfeh, "A numerical study of the rheological properties of suspensions of rigid, non-Brownian fibres,” J. Fluid Mech. 329, 155-186 (1996).

Mewis, J., and A. B. Metzner, "The rheological properties of suspensions of fibres in Newtonian fluids subjected to extensional deformations," J. Fluid Mech. 62, 593-600 (1974).

Meyer, R., and D. Wahren, "On the elastic properties of three-dimensional fibre networks," Sven. Papperstidn. 67, 432-436 (1964).

Milliken, W. J., M. Gottlieb, A. L. Graham, L. A. Mondy, and R. L. Powell, "The viscosity volume fraction relation for suspensions of rod-like particles by falling ball rheometry," J. Fluid Mech. 202, 217-232 (1989).

Nawab, M. A., and S. G. Mason, "The viscosity of dilute suspensions of thread-like particles," J. Phys. Chem. 62, 1248-1253 (1958)

Petrich, M. P., D. L. Koch, and C. Cohen, "An experimental determination of the stress-microstructure relationship in semi-concentrated fiber suspensions," J. Non-Newtonian Fluid Mech. 95, 101-133 (2000).

Ross, R. F., and D. J. Klingenberg, "Dynamic simulation of flexible fibers composed of linked rigid bodies," J. Chem. Phys. 106, 2949-2960 (1997).

Schmid, C. F., L. H. Switzer, and D. J. Klingenberg, "Simulations of fiber flocculation: Effects of fiber properties and interfiber friction," J. Rheol. 44, 781-809 (2000).

Soszynski, R. M., and R. J. Kerekes, "Elastic interlocking of nylon fibers suspended in liquid. Part 1. Nature of cohesion among fibers,” Nord. Pulp Pap. Res. J. 4, 172-179 (1988).

Sundararajakumar, R. R., and D. L. Koch, "Structure and properties of sheared fiber suspensions with mechanical contacts," J. Non-Newtonian Fluid Mech. 73, 205-239 (1997).

Switzer III, L. H., Simulating Systems of Flexible Fibers, Ph.D. thesis, University of Wisconsin-Madison, Madison, WI (2002).

Thalén, N., and D. Wahren, "Shear modulus and ultimate shear strength of some paper pulp fibre networks," Sven. Papperstidn. 67, 259-264 (1964).

Wahren, D., "On the visco-elastic properties of fibre networks," Sven. Papperstidn. 67, 378-381 (1964)

Wittenburg, J., Dynamics of Systems of Rigid Bodies (Teubner, Stuttgart, 1977).

Yamamoto, S., and T. Matsuoka, "A method for dynamic simulation of rigid and flexible fibers in a flow field," J. Chem. Phys. 98, 644-650 (1993).

Yamamoto, S., and T. Matsuoka, "Viscosity of dilute suspensions of rodlike particles: A numerical simulation method," J. Chem. Phys. 100, 3317-3324 (1994).

Yamane, Y., Y. Kaneda, and M. Doi, "Numerical simulation of semi-dilute suspensions of rodlike particles in shear flow," J. Non-Newtonian Fluid Mech. 54, 405-421 (1994). 